\title{
Evaluation of the modern luque trolley construct for treatment of early onset scoliosis using a gliding implant in an immature animal model
}

\author{
Ouellet, Jean A ; Ferland, Catherine E ; Racloz, Guillaume ; Klein, Karina ; Richter, Henning ; Steffen, Thomas ;
} von Rechenberg, Brigitte

\begin{abstract}
STUDY DESIGN: Experimental animal study. OBJECTIVE: To determine biological compatibility, stability, and growth potential of the Trolley Gliding Vehicle (TGV) used in a novel surgical technique for guided spinal growth. SUMMARY OF BACKGROUND DATA: Current treatments for Early Onset Scoliosis (EOS) maintaining spinal growth consist of posteriorly based spinal constructs requiring repetitive lengthening. Such interventions have a high rate of complications. Using a muscle sparing technique, a modified dual growing rods construct and new sliding spinal anchors, we set out to test a Modern Luque Trolley construct in an immature animal model. METHODS: Six matched pairs of 3 month old lambs were randomized to an observation or surgical group and were followed for 9 months. The surgical group underwent implantation of a Modern Luque Trolley construct with the new TGV inserted in a minimally invasive transmuscular technique capturing the spine and the 2 overlapping rods on either side. Physical exams and imaging were conducted at routine intervals, with a subsequent necropsy. RESULTS: The spines of the study group grew $96 \%$ between the instrumented segments compared to the control group without evidence of implant failure. Forty-two \% of the fixed anchors (pedicle screws) and $13.90 \%$ of the TGV were loose. All 6 animals had some heterotrophic bone formation tracking along the rods $(<20 \%)$ mainly originating from the distal anchor point. We identified 19 unplanned spontaneous facet arthrodesis out of the 132 mobile facets found between the fixed proximal and distal anchors. An additional 10 facets spontaneously fused proximal to the most proximal instrumented implants. CONCLUSIONS: Implantation of a Modern Luque construct with Trolley gliding vehicle allows for spinal growth in a non-scoliotic animal model. Implant loosening was likely mechanical as no sign of reactive inflammatory reaction were found. Reducing heterotrophic ossification and spontaneous facet arthrodesis remains a challenge in managing immature spine.
\end{abstract}

DOI: https://doi.org/10.1097/BSD.0000000000000260

Posted at the Zurich Open Repository and Archive, University of Zurich

ZORA URL: https://doi.org/10.5167/uzh-122736

Journal Article

Accepted Version

Originally published at:

Ouellet, Jean A; Ferland, Catherine E; Racloz, Guillaume; Klein, Karina; Richter, Henning; Steffen, Thomas; von Rechenberg, Brigitte (2017). Evaluation of the modern luque trolley construct for treatment of early onset scoliosis using a gliding implant in an immature animal model. Journal of Spinal Disorders Techniques, 30(40):E460E465.

DOI: https://doi.org/10.1097/BSD.0000000000000260 
(a) Evaluation of the Modern Luque Trolley Construct for Treatment of Early Onset Scoliosis Using a Gliding Implant in an Immature Animal Model

(b) Jean A Ouellet, MD FRCSC ${ }^{1}$, Catherine E Ferland, $\mathrm{PhD}^{1}$, Guillaume Racloz, $\mathrm{MD}^{2}$, Karina Klein, $\mathrm{VMD}^{3}$, Henning Richter, $\mathrm{VMD}^{3}$, Thomas Steffen, $\mathrm{PhD}^{4}$, Brigitte von Rechenberg, $\mathrm{VMD}^{5}$

${ }^{1}$ McGill Scoliosis \& Spine Centre, McGill University Health Centre, Montreal, Canada

${ }^{2}$ Orthopaedic Surgery Service, Geneva University Hospitals \& Medical School, University of Geneva, Geneva, Switzerland

${ }^{3}$ Musculoskeletal Research Unit (MSRU), Equine Department, University of Zurich, Switzerland

${ }^{4}$ Orthopaedic Research Lab, McGill University Health Centre, Montreal, Canada

${ }^{5}$ Competence Center for Applied Biotechnology and Molecular Medicine (CABMM), Equine Department, University of Zurich, Switzerland

(c) Address of correspondence and reprint requests to:

Dr. Jean A. Ouellet, MD, FRCSC

Montreal Children Hospital Chief of Scoliosis \& Spine Center

Copyright (C) 2015 Wolters Kluwer Health, Inc. Unauthorized reproduction of the article is prohibited. 
2300 Tupper, C521 McGill University Health Centre

Montreal, H3H 2B1 CANADA

Tel: 1514412 4464, Fax: 15144124353

Email: jean.ouellet@muhc.mcgill.ca

(e) This research was funded by industry, Synthes.

(f) Conflict of Interest: JAO is a consultant for DepuySynthes for the development of growing rod technology and receives no royalties. His Institution receives research support from the AO Foundation, AO North America and the Shriners Hospitals for Children. BVR has received research support from Synthes. CEF has received Fellowship support from the Shriners Hospitals for Children. For the remaining authors, none were declared.

Copyright (C) 2015 Wolters Kluwer Health, Inc. Unauthorized reproduction of the article is prohibited. 


\section{ABSTRACT}

Study design: Experimental animal study.

Objective: To determine biological compatibility, stability, and growth potential of the Trolley Gliding Vehicle (TGV) used in a novel surgical technique for guided spinal growth.

Summary of Background Data: Current treatments for Early Onset Scoliosis (EOS) maintaining spinal growth consist of posteriorly based spinal constructs requiring repetitive lengthening. Such interventions have a high rate of complications. Using a muscle sparing technique, a modified dual growing rods construct and new sliding spinal anchors, we set out to test a Modern Luque Trolley construct in an immature animal model.

Methods: Six matched pairs of 3 month old lambs were randomized to an observation or surgical group and were followed for 9 months. The surgical group underwent implantation of a Modern Luque Trolley construct with the new TGV inserted in a minimally invasive transmuscular technique capturing the spine and the 2 overlapping rods on either side. Physical exams and imaging were conducted at routine intervals, with a subsequent necropsy.

Results: The spines of the study group grew $96 \%$ between the instrumented segments compared to the control group without evidence of implant failure. Forty-two \% of the fixed anchors (pedicle screws) and $13.90 \%$ of the TGV were loose. All 6 animals had some heterotrophic bone formation tracking along the rods $(<20 \%)$ mainly originating from the distal anchor point. We identified 19 unplanned spontaneous facet arthrodesis out of the 132 mobile facets found between the fixed proximal and distal anchors. An additional 10 facets spontaneously fused proximal to the most proximal instrumented implants.

Conclusions: Implantation of a Modern Luque construct with Trolley gliding vehicle allows for spinal growth in a non-scoliotic animal model. Implant loosening was likely mechanical as no 
sign of reactive inflammatory reaction were found. Reducing heterotrophic ossification and spontaneous facet arthrodesis remains a challenge in managing immature spine.

Keywords: Early Onset Scoliosis; growth guidance; growing rod; Modern Luque Trolley; posterior spinal instrumentation; gliding vehicle; growth modulation; animal model

Copyright (C) 2015 Wolters Kluwer Health, Inc. Unauthorized reproduction of the article is prohibited. 


\section{INTRODUCTION}

The challenge of managing early-onset scoliosis (EOS) is the prevention of curve progression, while maintaining spinal growth (1-4). As it stands, current treatment options for EOS consist of serial casting and bracing (5). Once conservative treatments have failed, then posteriorly based spinal constructs are inserted in the hopes to control the curves (6). Unfortunately, both the spinal based (Dual Growing Rods) and the rib based (Vertical Expandable Prosthetic Titanium Ribs) construct requires repetitive surgeries at 4-6 month intervals allowing for spinal growth (2, 3, 7-9). Newer technologies using magnetically driven mechanisms are currently being tested (10). Alternative approaches to avoid repetitive surgeries consist of growth guidance systems such as the old Luque Trolley $(11,12)$ or the Shilla procedure $(2,13)$ (Figure 1). Both of these techniques rely on gliding spinal anchors allowing the spine to grow along fixed rods. The difference between these two constructs is that the Shilla procedure fixes one pair of rods at the apex of the curve and the proximal and distal spine glides apart from its apical anchor (13), while the Modern Trolley construct fixes one pair of rods proximally and one pair of rods distally with apical gliding anchors capturing all four overlapping rods (14). As the spine grows the proximal and distal fixed anchors grow apart while still capturing the apical gliding anchors.

The classic Luque Trolley technique as described by Eduardo Luque has lead to a high rate of implant failures, spontaneous fusion, and poor deformity control, resulting in alternative procedures for the management of EOS $(15,16)$. As implant technology has evolved and the shortcomings of the Luque Trolley have been understood better, a new surgical technique has been developed and takes advantage of a muscle sparing surgical approach $(13,14)$. A new

gliding spinal anchor, Trolley Gliding Vehicle (TGV)® (DepuySynthes Spine, Switzerland) has 
been developed to minimize the rate of implant failure. The implant consists of a dual core threaded pedicle screw coupled to a peak cable tie lined with a dual saddle polyethylene spacer. As the cable tie is tightened, the paired highly polished $5 \mathrm{~mm}$ titanium bullet nosed rods are captured and seated side by side, still allowing the rods to glide (Figure 2).

The purpose of this research was to investigate the biological impact of implanting a growth guidance system using a muscle sparing approach with the newly developed gliding spinal anchors in a growing animal model.

\section{MATERIALS AND METHODS}

All experiments were conducted according to Animal Welfare and permission was granted by the local federal authorities (application \# 181/2009). Twelve skeletally immature (3 month old) male Swiss alpine sheep were used for this study. To remove bias as to variation in sheep size and age, we paired the sheep by matching them for size, weight and age. Then, we randomly assigned one of the paired sheep to either the control group or the study group and followed them for 9 months. The control group had no surgical intervention done. The study group had threemidline incisions spanning the three segments of the spine to be instrumented: T3-T4; T8, T10, T12; L3-L4. A standardized construct was created spanning the proximal thoracic spine down to the high lumbar segments. The proximal and distal fixed anchor consisted of two sets of standard pedicle screws (USS II®, Depuy Synthes Spine, Switzerland) inserted into two adjacent vertebrae (T3-T4 and L3-L4). Three middle levels were skipped and followed by three pairs of TGV inserted at alternative levels (T8, T10, T12). The resulting construct had a total of 11 pairs of mobile facet joints between the proximal and distal anchors. At the fixed proximal and distal anchorage points, a classic subperiosteal dissection was performed as these segments were fused, 
using local bone graft augmented with chronOS Strip ${ }^{\circledR}$ (Depuy Synthes Spine, Switzerland). Via the middle incision using a paralateral approach, TGV were inserted with a freehand technique into the pedicle. A transmuscular dissection, lateral to the midline erector spinae was performed directly onto the transverse process avoiding the lamina and the facets, leaving muscles and ligaments intact across the midline spine. The dissection for these gliding anchors was kept to a minimum to avoid exposure of the bone or periosteum (Figure 3). Via the proximal incision (T3T4), two identical $5 \mathrm{~mm}$ rods were inserted, one on either side of the spinous processes, and tunneled in a submuscular fashion down past the T8, T10 and T12 gliding pedicle screws until L2. The rods were fixed tightly with the usual collar and nuts at the pedicle screws T3, T4. Via the distal incision at L3-L4, two identical rods were inserted, one on either side of the spinous processes, and tunneled upwards in a submuscular fashion passing the T8, T10 and T12 gliding vehicle until T5. At the T8, T10 and T12 levels, the TGV captured the rods using the cable ties. The ties were tightened ensuring the rods were parallel (Figure 4). Once the construct was completed, the soft tissues closure was performed in a standard fashion.

All 12 animals were examined 3, 6 and 9 months to inquire for the presence of deformity, tenderness, masses or evidence of neurological deficit. Imaging modalities consisting of plane radiographs and computed-tomography (CT) scans were performed at 3,6 and 9 months and post-euthanasia. The six surgical animals and 2 control animals were euthanized after the 9month observation.

Spinal growth was measured by acquiring true sagittal images off of the CT-scans (Figure 5). Different segments of the spine were then measured allowing monitoring of differential growth across different instrumented and non-instrumented segments of the spine (Figure 6). Lengths of the segments were measured twice by two independent reviewers, and means were reported in 
centimeters using 2 decimal points. Percentage of growth was calculated by measuring the Defined segment at Time X - Defined Segment at Time 0, divided by Defined Segment at Time 0 multiplied by 100 .

The spine was harvested, including the entire instrumented segment plus one vertebra above and below. The presence of facet arthrodesis was assessed and documented (Figure 7) by CT scan, and manual testing of facets mobility was performed once the spine was explanted. No abnormalities of the spine were found for the two control animals. The whole spine was examined macroscopically to document any abnormal soft tissue reactions around the spinal implants. Particular attention was paid to the soft tissue around the TGV looking for granulomas and reactive tissue. Juxta-positional soft tissues directly above the screws were histologically analyzed. The extracted spines were radiographed after removal of the rods. Macroscopic findings such as evidence of spontaneous heterotrophic bone formation, hardware failure, and/or screw loosening were documented.

A detailed histological evaluation of the facets, discs, growth plates and surrounding soft tissues was performed on selected ground sections samples (Figure 8). On a cellular level, a pragmatic approach was used to confirm our CT imaging findings. Any segment, with abnormalities found on imaging had detailed histological analysis performed. The corresponding normal control segments were also analyzed for direct comparison. Randomly chosen normal looking segments (e.g. with no apparent pathology) in the surgical group were also evaluated to identify any additional adverse event. In total, $80 \%$ of the segments were histologically assessed. Hence, current histological results provide a good general tendency but no statistical analysis could be generated. In addition, microradiographs of the segments histologically analyzed were obtained and compared to the CT imaging results. 
The occurrence of spinal fusion in the sections was scored in a semi-quantitative analysis described in Table 1. The soft tissues harvested around the TGV were analyzed for the cellular reaction and tissue scarring, including wear debris particles of the polyethylene with Light microscopy. A semi-quantitative histological evaluation using a scoring system was performed for microscopic cell counts of macrophages, mononuclear cells (lymphocytes, plasmocytes and monocytes) and fibroblasts (in the granulation tissue), as described in Table 1.

Copyright (C) 2015 Wolters Kluwer Health, Inc. Unauthorized reproduction of the article is prohibited. 


\section{RESULTS}

The modern Luque Trolley self-growing rod constructs were successfully implanted in all six immature animals without any perioperative complications. Throughout the 9-month observation period, all 6 surgical sheep behaved and roamed identically to the 6 control animals.

The matching CT-evaluation revealed to be effective as our groups had similar spinal lengths at time zero. The surgical group had an average spinal length across the instrumented spine of 32.5 $\mathrm{cm}$ while the observation group had a spinal length of $32.2 \mathrm{~cm}$. At the 9-month follow-up, the surgical group had grown, on average, 17\% across the instrumented segments T3 to L3, representing $5.6 \mathrm{~cm}$, while the control group had grown across the similar segments $6.6 \mathrm{~cm}$, representing $20 \%$ of their initial average length at time zero. This $3 \%$ difference of growth is explained by the planned fusion across the two proximal and distal fused segments for the proximal and distal fixed anchors. If one excludes the planned fused segments, i.e. the growth between the proximal and distal fixed anchors ( $T 5$ to L1), the spines grew on average $4.6 \mathrm{~cm}$ in the surgical group representing $21 \%$ of its original length while the control group grew $4.9 \mathrm{~cm}$ for the same segments representing $22 \%$ of their original lengths. Based on the CT data, the modern Luque Trolley system allows the instrumented segment of the spine to grow approximately $96 \%$ of the total length of a non-instrumented spine.

Looking at heterotrophic bone formation, all six instrumented animals showed heterotrophic bone along short segments of their rods, representing less than $20 \%$ of the entire length of the rods. In two of these animals $(5107,5108)$ the heterotrophic bone was part of a spontaneous fusion (bilateral facet arthrodesis) cranial to the caudal anchors (L2-L3). Only one animal (5110) 
had heterotrophic bone formation at the level of a Trolley screw (T6, right side) travelling cranially and caudally. This sheep developed broad ossification on either side of the spine.

Looking specifically at spontaneous facet arthrodesis, all 26 planned fused facet joints of the cranial and caudal fix anchors fused as expected (Figure 8, segment 4), while an additional 29 facet joints fused spontaneously. Ten of these additional fused facets were cranial and bilateral to the proximal anchor, which resulted in an additional fused segment in five animals $(5105,5107$, $5108,5109,5110)$ cranial to the instrumented spine. The other 19 spontaneously fused facets were between the fixed cranial and caudal anchors, resulting in a $14 \%$ spontaneous fusion rate (19 out of 132 mobile facets across the instrumented spine). Five animals $(5105,5106,5107$, 5108,5109 ) showed a spontaneously fused segment (bilateral facet arthrodesis) just cranial to the fixed caudal anchor, representing 10 of the 19 fused facets in between the instrumented area. The remaining 9 facet arthrodeses were detected cranially to a TGV (Figure 8, segment 2): Four animals ( 8 facets) had bilateral facet arthrodesis cranial to a TGV (5106 T11-T12, 5108 T10T11, 5109 T11-T12, 5110 T10-T11) and one animal had a unilateral facet arthrodesis cranial to a TGV (5107 T10-T11). Overall, these spontaneous facet arthrodeses resulted in an additional unplanned two level fusion in 5 of 6 animals across the instrumented spine.

All spinal segments with abnormalities found on plain x-ray and/or CT scan were histologically analyzed. For comparison, their corresponding normal control segments were also evaluated histologically. The randomly chosen normal looking segments which were analyzed demonstrated absence of abnormal findings. All macroscopic and CT imaging findings were confirmed by microradiographs and histology. At necropsy, there was no evidence of implant failure. However, it was apparent that half of the animals had various loose screws. With manual testing and using plain radiographs it was found that $42 \%$ (20 of 46 ) of the pedicle screws were 
loose, all of which were at the caudal fixed anchor point while only $13.90 \%$ (5 of 36) of the TGV were loose. This bone screw interface loosening was confirmed by microradiographs and histology (Figure 8, segment 1). Neither granulomas were found locally surrounding the implants nor was there systemic evidence of particle polyethylene or peak debris around the TGV. Titanium debris was found in two animals where there was evidence of rod on rod friction. Evaluation of paraffin sections above the TGV revealed only few, singular and negligible polyethylene particles such that the statistical evaluation and grading could not be performed.

\section{DISCUSSION}

In this study, the biological impact of implanting a growth guidance system using a muscle sparing approach with the newly developed gliding spinal anchors was assessed in a growing animal model. Lamb spines have similar morphometric dimensions as a typical spine of a child with EOS. Moreover, sheep have a high predilection to spontaneous fusion similar to young children, thus making it the best-suited animal model to investigate growth guidance systems.

The gliding spinal anchors evaluated in this study demonstrated high potential for selflengthening as a treatment option for EOS. The results confirm that without additional lengthening procedures, a modern Luque Trolley construct allows for a 96\% spinal growth to that of non-instrumented non-scoliotic sheep spines. The slightly retarded growth was attributed primarily to the fusion of the proximal and distal anchored segments but not to the dynamic segments containing the TGV. This growth occurred despite the evidence of spontaneous facet arthrodesis adjacent to the proximal and distal fixed pedicle and partial heterotopic bone formation along the rods. A possible explanation as to the maintenance of growth is that these spontaneous fusion and heterotopic bone are not solid enough to inhibit vertebral growth. 
Interestingly, the vertebrae maintained their height comparable to those of the control group, in contrast to the discs at the levels of the planned or spontaneous facet fusion, which lost some of their height, thus explaining the difference between the study and control groups. It is thought that the subperiosteal dissection, the muscle stripping and the repetitive muscle contusion by the rods triggered this spontaneous heterotopic bone formation originating from the fixed anchors. In contrast at the level of the transmuscular, lateral para-spinal minimal invasive approach, only one animal had heterotrophic bone formation along its rod, and had few spontaneous facet arthrodesis. A nine-month follow-up is a relatively short follow-up period for growth sparing surgery and further spontaneous fusions or additional heterotrophic bone formation may occur over an extended period of time. Such findings match clinical findings when growth sparing surgeries are converted to final fusion with evidence of partial fusion across the growing constructs. Spontaneous fusion and/or heterotrophic complications remain one "drawback" of posteriorly based growing rod constructs. Granted that the current goal of spine deformity management is a solid spinal fusion, our ultimate hope is that we can develop a growing rod construct that will not result in spontaneous fusion.

Comparative research on posterior growth guidance system in animals is limited to McCarthy et al. publication on a caprine animal model testing the posteriorly based Shilla Growing Rods construct (17). They implanted eleven immature goats and followed them for 6 months. Our results are consistent with their findings as to ongoing growth. However, they did not quantify the percentage of growth maintained, as they did not have a control group. They also noted alter facets morphology next to the Shilla screws though did not find as we did spontaneous facet arthrodesis. In contrast to our results they noted significant soft tissue reaction surrounding the Shilla screws with metal filings adjacent to the gliding screws as well as in the para-aortic lymph 
nodes. Histological studies showed phagocytic uptake of the metal fragments. They did not mention the presence or absence of implant loosening.

Our results did show that the Pedicle screws were loosened more so in the fused proximal segments and much less in the dynamic TGV in the middle segment. Although not tested mechanically, this phenomenon can be explained based on the levels we chose to instrument, and the lambs' necessity to maintain good neck motion. Nevertheless, from a histological point of view, the loosening was in keeping with mechanical loosening with fibrous ingrowth around the pedicle screws (membranous interface) rather than an inflammatory response. The tissue consisted mainly of scar tissue with a physiological reaction to constant micromotion. Polyethylene wear debris particles in the soft tissue surrounding the Trolley screws were present in low concentrations and not accompanied by foreign body reactions indicating biocompatibility problems.

In conclusion, the findings confirm that Modern Luque Trolley construct with Trolley gliding vehicles allow for spinal growth in a non-scoliotic animal model over a 9-month period. Implant loosening occurred as a consequence of micromotion rather than of tissue reaction to the implant. Heterotrophic ossification and spontaneous arthrodesis remain a challenge in immature spines. Surgical breach of the proximal facet joint is a predisposition to spontaneous arthrodesis even when using a transmuscular approach. The assessment of the Trolley to provide similar results in a deformed spine needs further investigation.

\section{Acknowledgment}

The authors would like to thank Mrs Guylaine Bédard for the artwork. 


\section{REFERENCES}

1. Gillingham BL, Fan RA, Akbarnia BA. Early onset idiopathic scoliosis. J Am Acad Orthop Surg. 2006 Feb;14(2):101-12.

2. Tis JE, Karlin LI, Akbarnia BA, et al. Early onset scoliosis: modern treatment and results. $J$ Pediatr Orthop. 2012 Oct-Nov;32(7):647-57.

3. Thompson GH, Lenke LG, Akbarnia BA, et al. Early onset scoliosis: future directions. J Bone Joint Surg Am. 2007 Feb;89 Suppl 1:163-6.

4. Akbarnia BA. Management themes in early onset scoliosis. J Bone Joint Surg Am. 2007. Feb;89 Suppl 1:42-54.

5. D'Astous J, Sanders J. Casting and traction treatment methods for scoliosis. The Orthop Clin North Am. 2007;38(4):477

6. Gomez JA, Lee JK, Kim PD, et al. "Growth friendly" spine surgery: management options for the young child with scoliosis. J Am Acad Orthop Surg. 2011 Dec;19(12):722-7.

7. Thompson GH1, Akbarnia BA, Campbell RM Jr. Growing rod techniques in early-onset scoliosis.J Pediatr Orthop. 2007 Apr-May;27(3):354-61.

8. Hasler CC, Mehrkens A, Hefti F. Efficacy and safety of VEPTR instrumentation for progressive spine deformities in young children without rib fusions. Eur. Spine J. 2010 Mar;19(3):400-8.

9. White KK, Song KM, Frost N, et al. VEPTR growing rods for early-onset neuromuscular scoliosis: feasible and effective. Clin Orthop Relat Res. 2011 May;469(5):1335-41.

Copyright (C) 2015 Wolters Kluwer Health, Inc. Unauthorized reproduction of the article is prohibited. 
10. Hickey BA, Towriss C, Baxter G, et al. Early experience of MAGEC magnetic growing rods in the treatment of early onset scoliosis. Eur Spine J. 2014 Apr;23 Suppl 1:61-5.

11. Luque ER, Cardoso A. Treatment of scoliosis without arthrodesis or external support, preliminary report. Orthop Trans 1977;1:37-38

12. Luque ER. Paralytic scoliosis in growing children, Clin Ortho Relat Res 1982;(163):202-209

13. McCarthy R.E, Luhmann S, Lenke L, et al. The Shilla growth guidance technique for earlyonset spinal deformities at 2-year follow-up: A preliminary report. J Pediatr Orthop. 2014 Jan;34(1):1-7

14. Ouellet JA. Surgical Technique: Modern Luque Trolley, a self-growing rod technique. Clin Orthop Relat Res 2011;469(5):1356-1367.

15. Mardjetko SM, Hammerberg KW, Lubicky JP, et al. The Luque trolley revisited. Review of nine cases requiring revision. Spine. 1992 May;17(5):582-9.

16. Pratt RK, Webb JK, Burwell RG, et al. Luque trolley and convex epiphysiodesis in the management of infantile and juvenile idiopathic scoliosis. Spine. 1999 Aug 1;24(15):1538-47.

17. McCarthy RE, Sucato D, Turner JL, et al. Shilla growing rods in a caprine animal model: a pilot study. Clin Orthop Relat Res. 2010 Mar;468(3):705-10.

Copyright (C) 2015 Wolters Kluwer Health, Inc. Unauthorized reproduction of the article is prohibited. 


\section{FIGURE LEGENDS}

Figure 1. Comparaison between Luque Trolley construct (A) and Shilla construct (B).

Figure 2. Trolley Gliding Vehicle design.

Figure 3. Transmuscular approach: Middle thoracic spine with 6 Trolley screws inserted via muscle sparing approach spanning 5 levels - Open cable tie with polyethylene liner showing. Figure 4. Final Modern Luque Trolley Construct implanted with proximal and distal fusion and middle gliding implants.

Figure 5. Three-dimentional acquisition of mid sagittal vertebra.

Figure 6. Heterotrophic bone formation at Trolley gliding vehicle 6 (right side) of sheep 5110 travelling cranially and caudally (right), and along / around the rods (left).

Figure 7. Imaging adverse sheet documentation identifying mobile and fused facets, altered disc height.

Figure 8. Microradiograph and histology imaging.

Copyright (C) 2015 Wolters Kluwer Health, Inc. Unauthorized reproduction of the article is prohibited. 


\begin{tabular}{|c|c|}
\hline Bony Bridging Across The Joint & Score \\
\hline $0 \%$ no bone & 0 \\
\hline $1-25 \%$ fibrous bridge & 1 \\
\hline $26-50 \%$ thin bony bridge & 2 \\
\hline$>50 \%$ thick bony bridge & 3 \\
\hline \multicolumn{2}{|l|}{ Percentage of Screw Osseointegration } \\
\hline $100 \%$ contact & 0 \\
\hline $1 / 2$ screw length $100 \%$ contact & 1 \\
\hline $1 \mathrm{~mm}$ of fibrous $>50 \%$ of screws & 2 \\
\hline$>1$ mm gap & 3 \\
\hline \multicolumn{2}{|l|}{ Width of Growth Plate } \\
\hline \multirow{2}{*}{\multicolumn{2}{|c|}{$\begin{array}{l}\text { Regular } \\
\text { Irregular }\end{array}$}} \\
\hline & \\
\hline \multicolumn{2}{|l|}{ Percentage of Columnar Cartilage Damage } \\
\hline $0 \%$ no change & 0 \\
\hline $\begin{array}{l}1-25 \% \text { altered height of cartilaginous } \\
\text { proliferative zone }\end{array}$ & 1 \\
\hline $26-50 \%$ marked altered proliferative zone & 3 \\
\hline$>50 \%$ angulated growth plates & \\
\hline \multicolumn{2}{|l|}{ Death of Nucleus Pulposus } \\
\hline No & 0 \\
\hline Yes & 1 \\
\hline
\end{tabular}

Copyright (C) 2015 Wolters Kluwer Health, Inc. Unauthorized reproduction of the article is prohibited. 


\begin{tabular}{|c|c|}
\hline \multicolumn{2}{|c|}{ Staining of Intervertebral Disc (Metaplasia) } \\
\hline Normal staining & 0 \\
\hline $1-25 \%$ deviation of staining & 1 \\
\hline $26-50 \%$ deviation of staining & 2 \\
\hline$>50 \%$ deviation of staining & 3 \\
\hline \multicolumn{2}{|l|}{ Cracks of Fibers in Disc } \\
\hline No cracks & 0 \\
\hline $1-35 \%$ cracks & 1 \\
\hline $26-50 \%$ cracks & 2 \\
\hline$>50 \%$ cracks & 3 \\
\hline \multicolumn{2}{|l|}{ Disc Height } \\
\hline \multicolumn{2}{|l|}{ Physiological } \\
\hline \multicolumn{2}{|l|}{$1-25 \%$ deviation } \\
\hline \multicolumn{2}{|l|}{$26-50 \%$ deviation } \\
\hline$>50 \%$ deviation & 3 \\
\hline \multicolumn{2}{|c|}{$\begin{array}{l}\text { Percentage of Inflammatory Cells in the Microscopi } \\
\text { Field of Sight }\end{array}$} \\
\hline $0 \%$ & 0 \\
\hline $1-25 \%$ & 1 \\
\hline $26-50 \%$ & 2 \\
\hline$>50 \%$ & 3 \\
\hline
\end{tabular}

Copyright (C) 2015 Wolters Kluwer Health, Inc. Unauthorized reproduction of the article is prohibited. 


\section{Modern Luque trolley Construct}

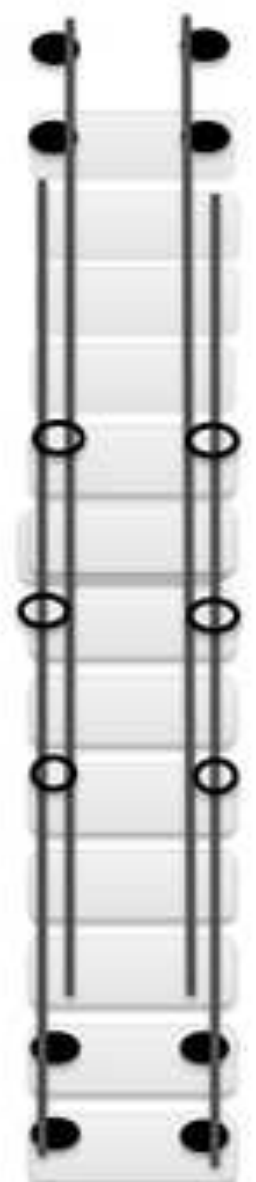

Shilla

Construct

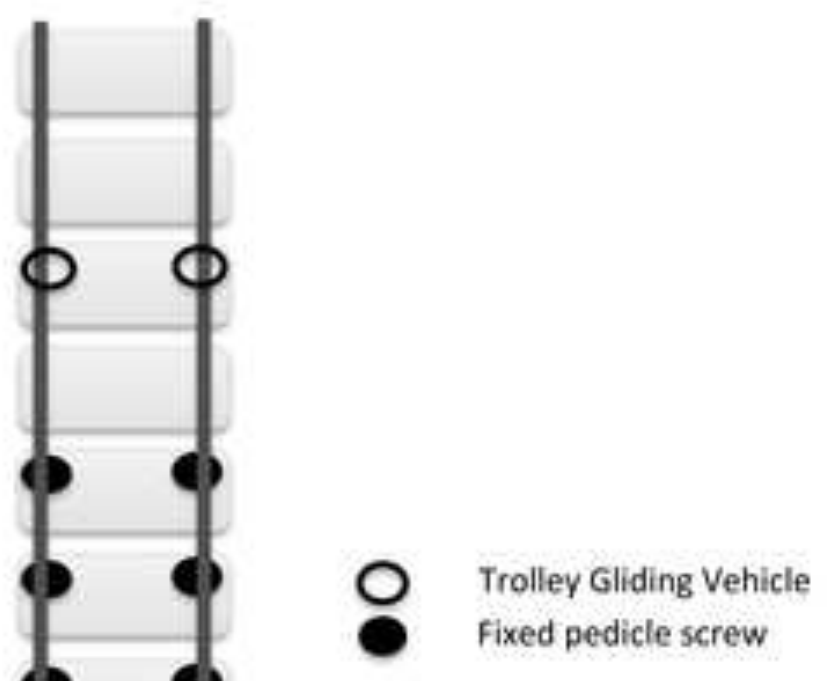


Click here to download high resolution image

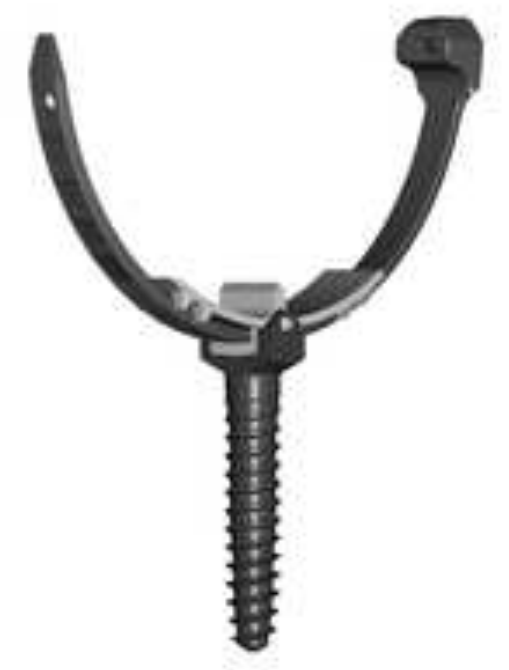

Copyright (C 2015 Wolters Kluwer Health, Inc. Unauthorized reproduction of the article is prohibited. 


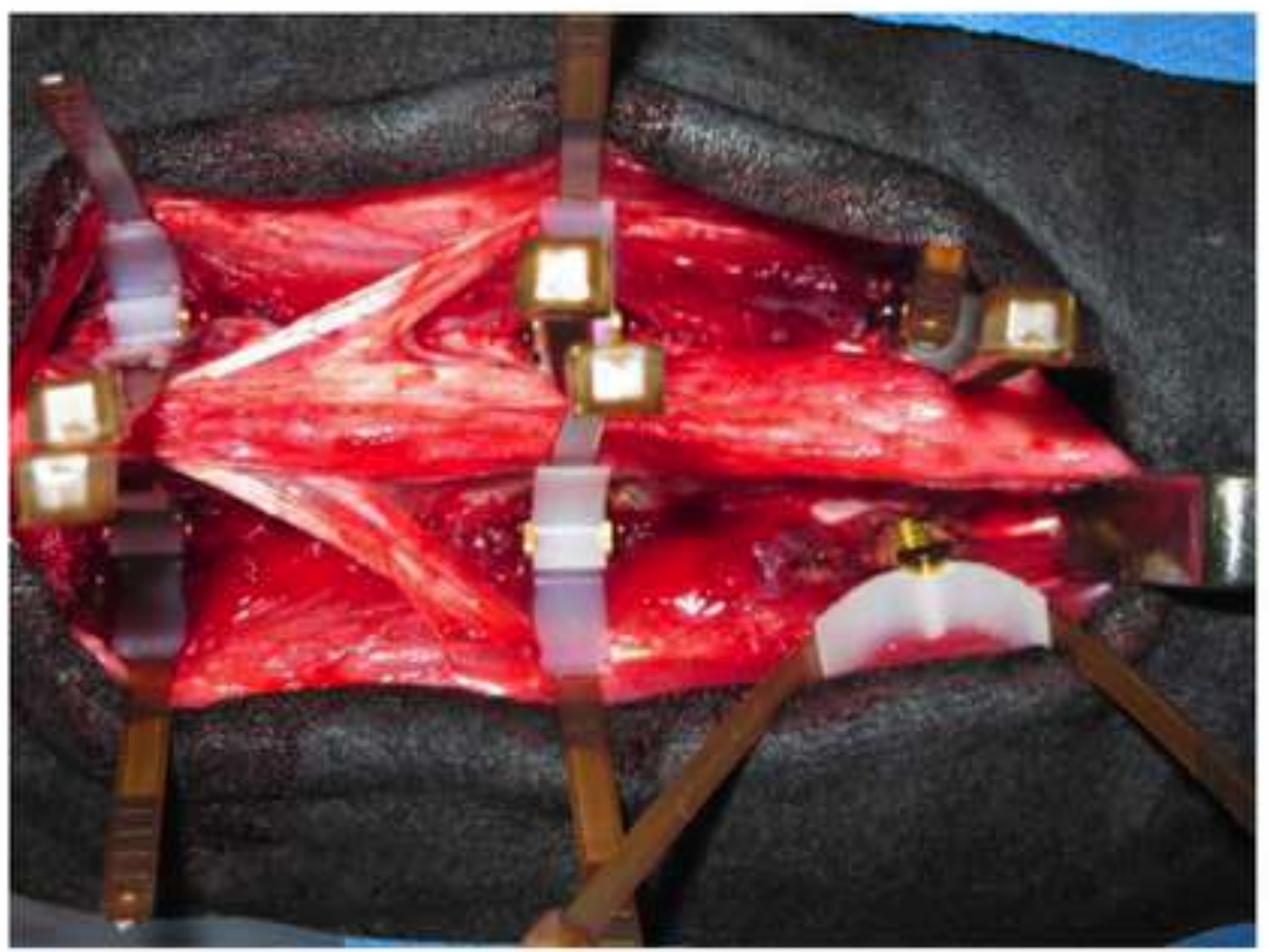

Copyright (C) 2015 Wolters Kluwer Health, Inc. Unauthorized reproduction of the article is prohibited. 
Click here to download high resolution image

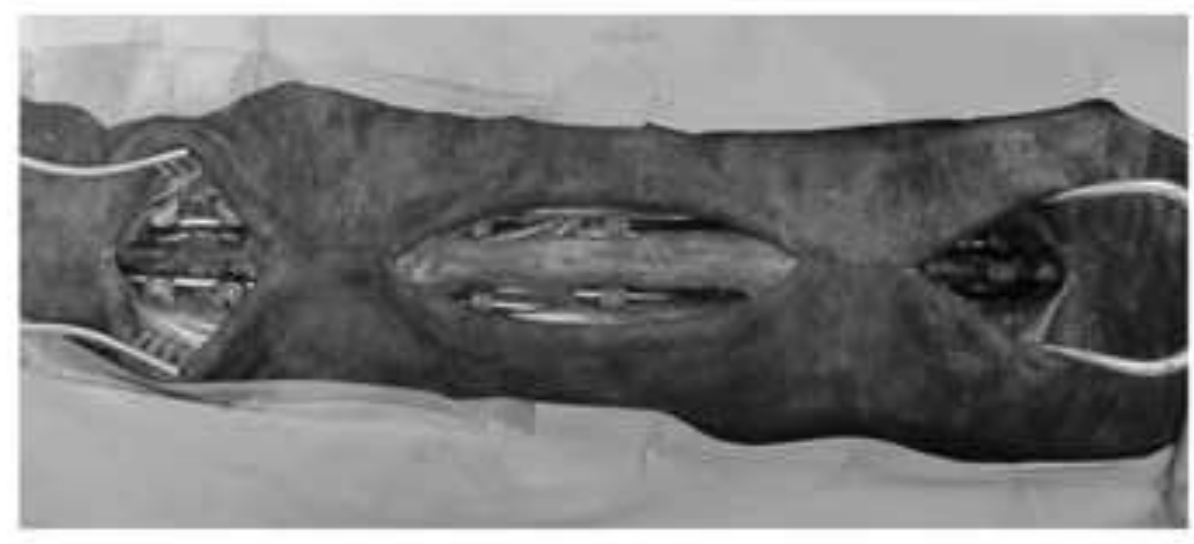

Copyright (C) 2015 Wolters Kluwer Health, Inc. Unauthorized reproduction of the article is prohibited. 


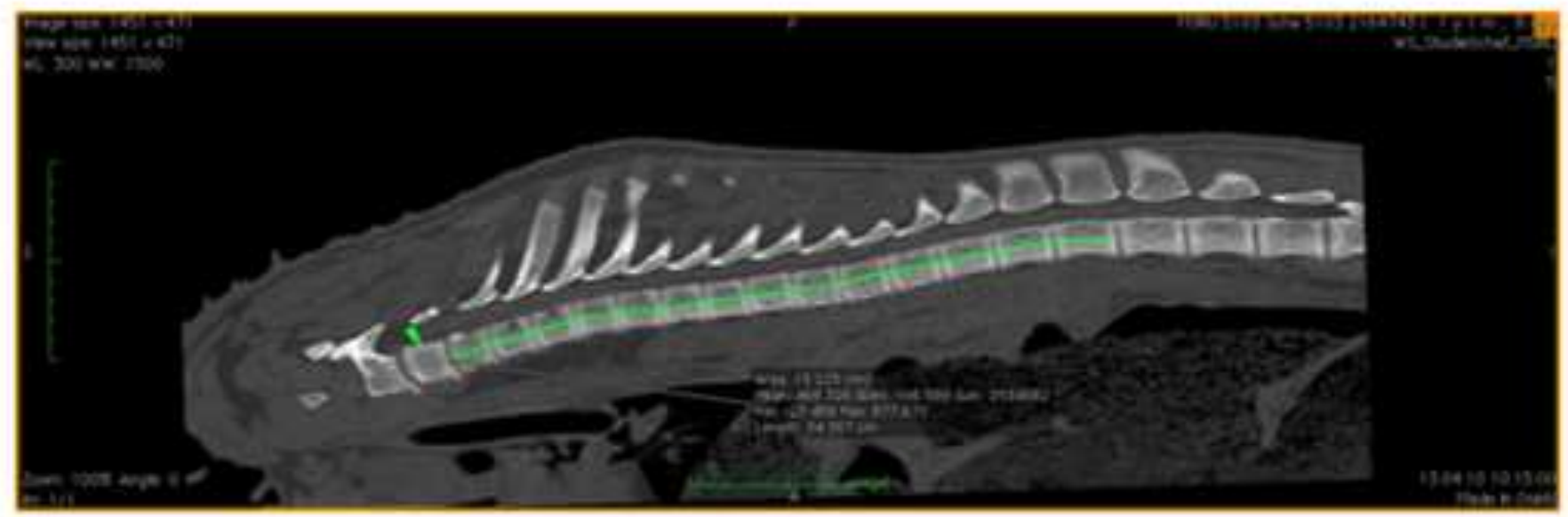

Copyright (C) 2015 Wolters Kluwer Health, Inc. Unauthorized reproduction of the article is prohibited. 


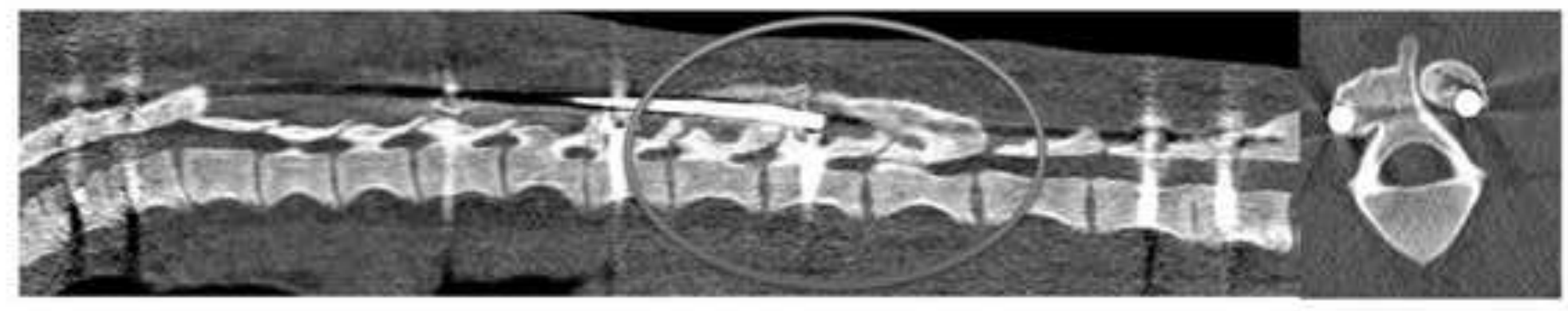

Copyright (C) 2015 Wolters Kluwer Health, Inc. Unauthorized reproduction of the article is prohibited. 


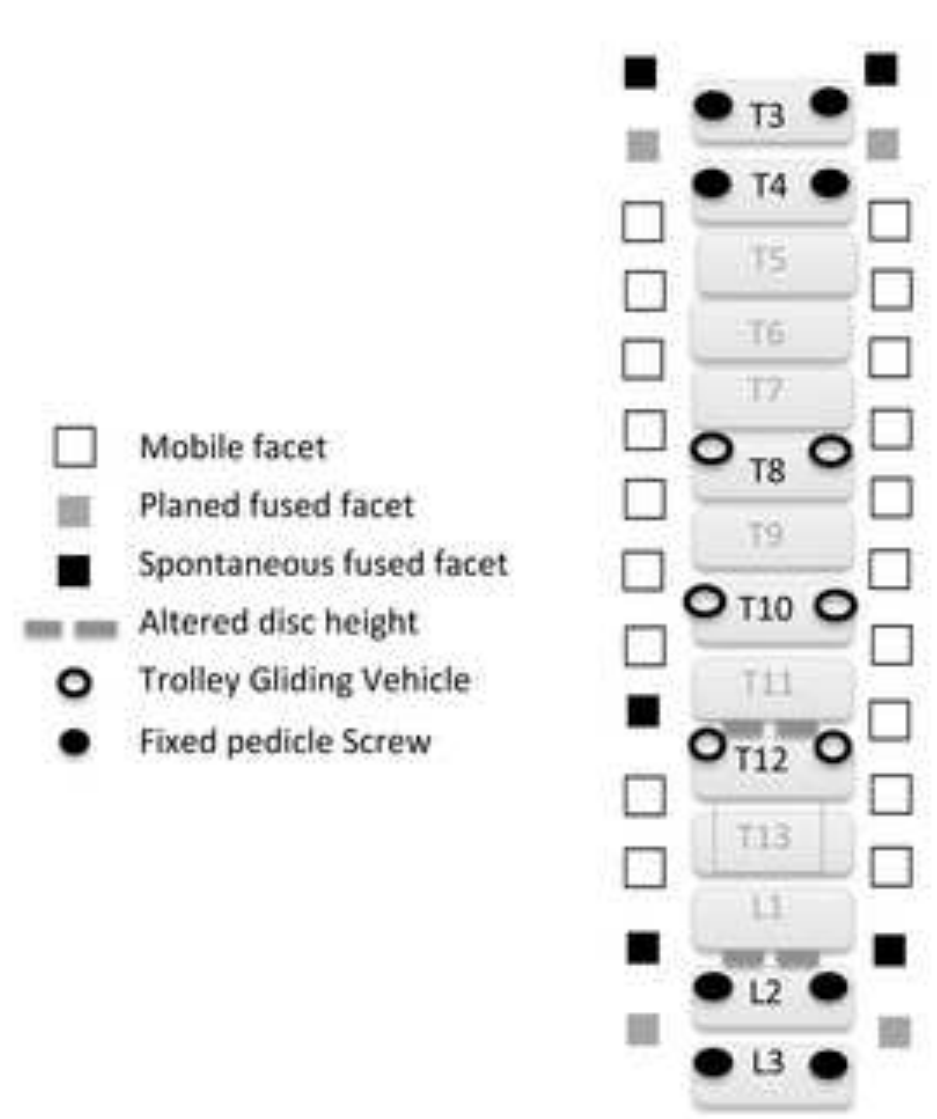

Copyright (C) 2015 Wolters Kluwer Health, Inc. Unauthorized reproduction of the article is prohibited. 


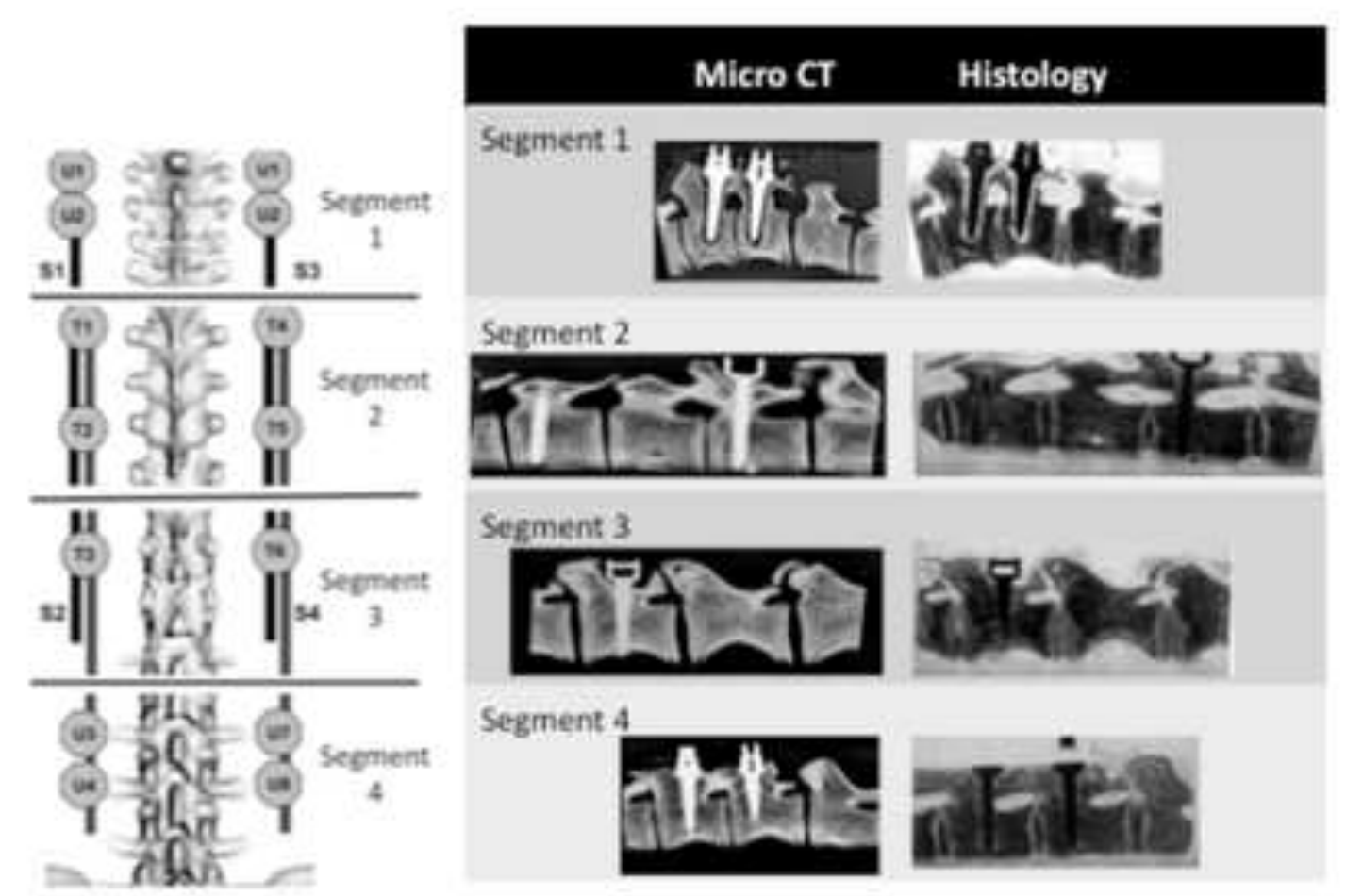

Copyright (C) 2015 Wolters Kluwer Health, Inc. Unauthorized reproduction of the article is prohibited. 